Frontiers in Heat and Mass Transfer

Editorial Announcement

\title{
FRONTIERS IN HEAT PIPES (FHP) IS MERGEED INTO FRONTIERS IN HEAT AND MASS TRANSFER (FHMT)
}

Frontiers Heat Pipes (FHP) is an international journal devoted mainly to the field of heat pipe science and technology. Since its inception in 2010, seven volumes have been published. Recent advances in heat pipe science and technology have demonstrated that heat pipes often function as an important part of a more complicated system that involves different modes of heat and mass transfer; further success of FHP and its impact are limited by the narrow scope of FHP. In order to broaden the impact of research in heat pipe science and technology, FHP is merged into its sister journal - Frontiers in Heat and Mass Transfer (FHMT) effective 2017. All papers published in FHP between 2010 and 2016 in Vol. 1-7 are permanently archived at the Thermal-Fluids Central website (http://thermalfluidscentral.org/journals/index.php/Heat_Pipes/issue/arc hive).

FHMT has enjoyed success and wide acceptance by the thermalfluids community since its inception in 2010. It is indexed by Scopus since 2012 and most recently it is indexed by the Web of Science. Research papers in heat pipe science and technology published in FHMT will undoubtedly gain more attentions from the community and have broader impact to all fields of science and engineering. We are looking forward to publish more high-quality research papers in heat pipe science and technology in FHMT.

Amir Faghri Founding Editor and Editor-in-Chief of FHP and FHMT Email: faghri@engr.uconn.edu

Yuwen Zhang Co-Editor-in-Chief of FHP and FHMT Email: zhangyu@missouri.edu 